\title{
Mechanical Chirality of Rotaxanes: Synthesis and Function
}

\author{
Kazuko Nakazono *(D) and Toshikazu Takata *(D)
}

School of Materials and Chemical Technology, Tokyo Institute of Technology, 4259 Nagatsuta-cho, Midori-ku, Yokohama, Kanagawa 226-8503, Japan

* Correspondence: nakazono.k.aa@m.titech.ac.jp (K.N.); takata.t.ab@m.titech.ac.jp (T.T.)

Received: 26 November 2019; Accepted: 7 January 2020; Published: 10 January 2020

\begin{abstract}
Mechanically chiral molecules have attracted considerable attention due to their property and function based on its unique interlocked structure. This review covers the recent advances in the synthesis and function of interlocked rotaxanes with mechanical chirality along with their dynamic and complex stereochemistry. The application of mechanically chiral rotaxanes to control the polymer helical structure is also introduced, where amplification of mechanical chirality appears to cause the macroscopic polymer property change, suggesting the potential applicability of mechanical chirality in polymer systems.
\end{abstract}

Keywords: mechanical chirality; rotaxane; mechanically interlocked molecules; chiral rotaxane; helical polymer

\section{Introduction}

Mechanically interlocked molecules (MIMs) consist of multiple molecular components entangled via mechanical bonds. Early research on MIMs started from the viewpoint of statistical synthesis and molecular topology, which has been followed by strategic synthesis based on host-guest chemistry and the active metal template method. Thus, catenanes and rotaxanes with various molecular structures have become presently available [1]. These molecules offer interesting properties that are based on the cooperative effect between their subcomponents, which can be altered at the mechanical bonds. Recently, various applications of the characteristic properties of MIMs have been actively studied by utilizing not only small molecules, but also polymer materials [2]. Although a variety of MIMs can be synthesized, research on the basic chirality of MIMs is still in its infancy. It is well known that chiral MIMs are obtained by introducing some classical chiral units to MIMs, whereas chirality also appears when combining $C_{\mathrm{s}}$ symmetrical components (Figure 1). Such chirality stems from the difference in the spatial arrangement of the components at the mechanical bond, and it is therefore called "mechanical chirality" or "topological chirality." Theoretical studies on this subject were reported in the 1960s [3-5]; thereafter, the first synthesis of mechanically chiral catenane and rotaxane was achieved in the late nineties by Sauvage et al. [6] and Vögtle et al. [7,8].

With the development of strategic synthetic methodologies, MIMs with different types of molecular chirality have been reported [1], and recently, the stereochemistry of MIMs has also been consolidated based on the concepts of "co-conformation" and "co-configuration" [9-12]. This stereoisomerism is generated not only by the orientation, sequence, and arrangement of multiple components, but also by their relative position. Moreover, the chirality arises from the combination of achiral components. These may or may not be convertible between the stereoisomers, depending on the mechanical bond movement; furthermore, it is sometimes possible to induce the switching between chiral and achiral structures. Therefore, the chirality of MIMs includes complex but interesting concepts, and special characteristics that cannot be found in conventional chiral molecules are expected. In the future, 
the variety of chiral MIMs derived by co-conformational changes could be used to create switchable molecular devices for various chiral applications. Thus, fundamental research on the chirality of MIMs is an important subject not only for basic science, but also for applications exploiting the nature of MIMs. Recently, some excellent reviews on chiral MIMs have been reported [13-15] that widely cover various compounds with chiral aspects. This review focuses on chiral rotaxanes and their applications directed toward an extensive analysis of mechanical chirality. In general, rotaxane can be synthesized easier than catenane, and has wide structural diversity, depending on the number and kind of components. Rotaxanes can exhibit some dynamic modes based on the intercomponent motions such as shuttling, circumrotation, and flipping around the mechanical bond, which leads to significant co-conformational changes and at times to a change in the chemical property of the functional groups in it [9]. In fact, a variety of molecular switches including a chiroptical switch system have been investigated by utilizing the rotaxane component's mobility. Not only have small molecules received considerable research attention, but also the polymeric systems possessing rotaxane moieties. The combination of polymer and rotaxane increases the structural and conformational diversity accompanied by the appropriate polymer property changes such as morphology or other physical properties according to the rotaxane's dynamic behavior. Therefore, we would like to focus on the potential of chiral rotaxane as a new chiral molecular motif.

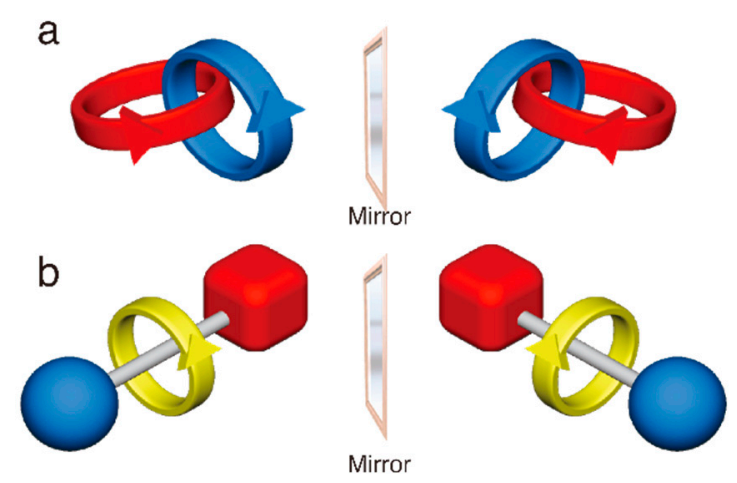

Figure 1. Mechanically chiral catenane (a) and rotaxane (b).

\section{Mechanically Chiral Rotaxanes}

Rotaxanes consist of macrocyclic components, so called wheels, threaded by axle components. As shown in Figure 2, the combination of unsymmetrical components inherently derives enantiomers.

Their chirality is related to the spatial arrangements of unsymmetrical component parts linked by a mechanical bond, and is different from the classical chirality based on covalent bonding. In fact, these enantiomers cannot be interconverted unless a mechanical bond breaks and is then re-formed. To express this characteristic chirality of mechanical bonded molecules, several terms have been proposed to properly represent this to date [16], but recently, an appropriate method has been established. Goldup proposed "mechanically planar chirality" as the more suitable description to express the stereochemistry of the rotaxane's molecular chirality [17]. To describe the chirality based on the spatial arrangement of components parts, he proposed adding " $m p$ " to the " $R$ " and " $S$ " as " $R_{\mathrm{mp}}$ " and "S $\mathrm{mp}_{\mathrm{m}}$ ". "mp" stands for mechanical planar, which means that the central plane of chirality corresponds to the plane including the wheel that is located at the vertical position to the axle component. As shown in Figure 2a, first the orientations of each component are defined based on the ordinary used priority rule, respectively. Then, the directions of the components are combined and determine the enantiomers as $R_{\mathrm{mp}}$ or $S_{\mathrm{mp}}$. On the other hand, the co-conformational change by moving the relative position of the wheel on the axle derives a different chiral stereoisomer. The co-conformational change, as shown in the bottom of Figure 2a, results in a drastic change in the spatial arrangement of component parts around the intersection of components, even though the orientations of the individual components do not change. It would not be surprising if such a co-conformational 
change resulted in the inversion of the circular dichroism (CD) signal. Such an apparent chiral inversion is significantly interesting from the view of chiroptical switching, but these co-conformers are not static, but dynamic, and usually have a structural distribution. If the isomerization barrier between these co-conformers is not high as to detect each co-conformer, it should be discussed by using the averaged structure based on the co-conformational distribution. In fact, when the rotaxanes are synthesized by utilizing local intramolecular interactions, the co-conformation is biased in a specific distribution even though the mechanical bond is movable. Whereas, when the intramolecular interaction of rotaxane is removed, a stable conformational distribution is spontaneously changed based on the updated potential gradient.

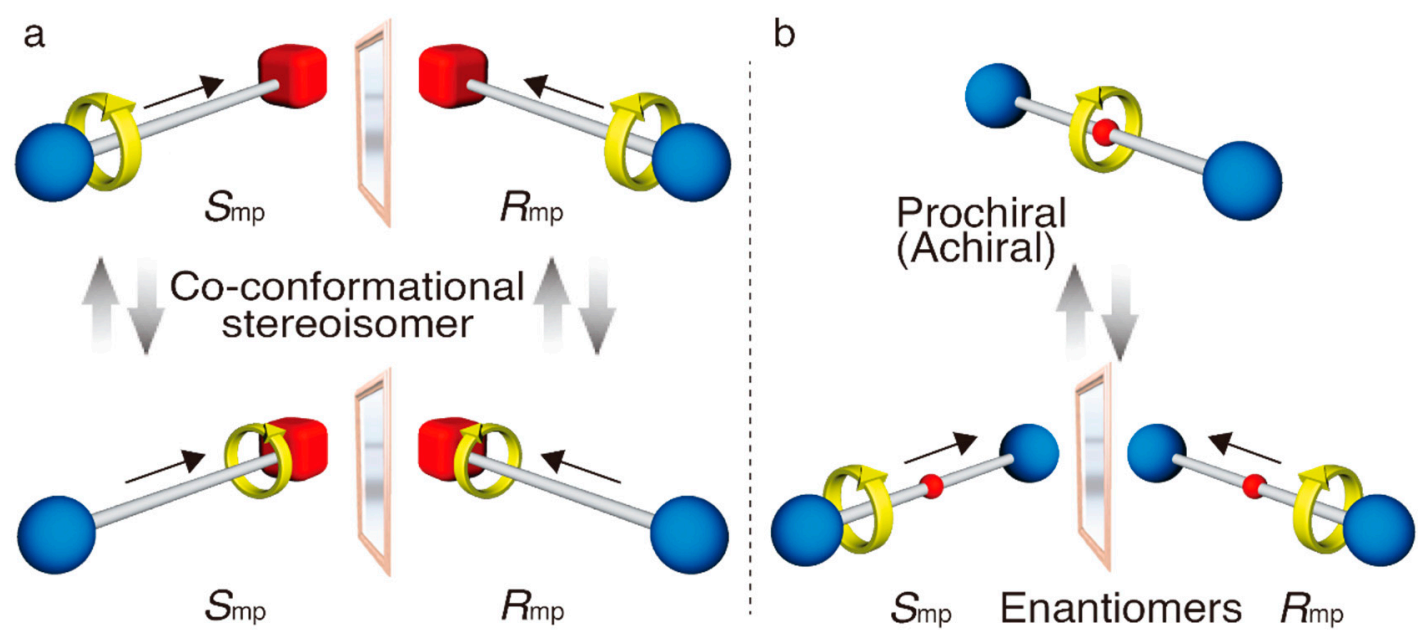

Figure 2. Mechanically planar chirality in rotaxane. Co-conformational stereoisomerization in [2]rotaxane having a rotationally unsymmetrical wheel and an axle with two nonequivalent ends (a), and achiral-chiral interconversion in [2] rotaxane having rotationally unsymmetrical wheel and an axle with two equivalent ends (b).

In addition to this, the dynamic stereoisomerization in a rotaxane means it is possible to control the achiral-chiral chiroptical switching in a [2]rotaxane, which consists of a symmetrical axle and unsymmetrical wheel (Figure 2b). Once the wheel moves from the central position of the axle, mechanically planar chirality is induced, depending on the co-conformation from achiral (prochiral). However, if the wheel can move freely on the axle (i.e., the racemization is too fast to detect each enantiomer), it can be regarded as an achiral molecule.

As above-mentioned, mechanical chirality is generated as the result of a static mechanostereoisomerism during the synthetic process, but it is possible to access various chiroptical states by their dynamic stereoisomerism property. From this point of view, mechanically chiral rotaxanes are an attractive chiral molecule with unique dynamic property.

\subsection{Mechanically Planar Chiral Rotaxanes: Stereochemistry, Synthesis, and Optical Resolution}

In 1997, Vögtle and Okamoto reported the first synthesis and HPLC optical resolution of mechanically chiral rotaxanes that consisted of an unsymmetrical axle and macrocyclic components (Figure 3a) [7,8]. They succeeded in isolating the enantiomerically pure [2] rotaxane $\left(S_{\mathrm{mp}} / \boldsymbol{R}_{\mathbf{m p}}\right)-\mathbf{1}$, followed by transforming it to [1]rotaxane $\left(S_{\mathrm{mp}} / \boldsymbol{R}_{\mathrm{mp}}\right)-\mathbf{1}^{\prime}$ by bridging the components via linking the sulfonamide moieties with an oligoethylene spacer (Figure $3 \mathrm{~b})$. The CD spectra of $\left(S_{\mathrm{mp}}\right) \mathbf{- 1}$ and $\left(\boldsymbol{R}_{\mathrm{mp}}\right) \mathbf{- 1}$ become a mirror image, and the enantiomeric relationship is also maintained after conversion to [1]rotaxane $\left(S_{\mathrm{mp}} / \boldsymbol{R}_{\mathrm{mp}}\right)-\mathbf{1}^{\prime}$, respectively. 
(a)
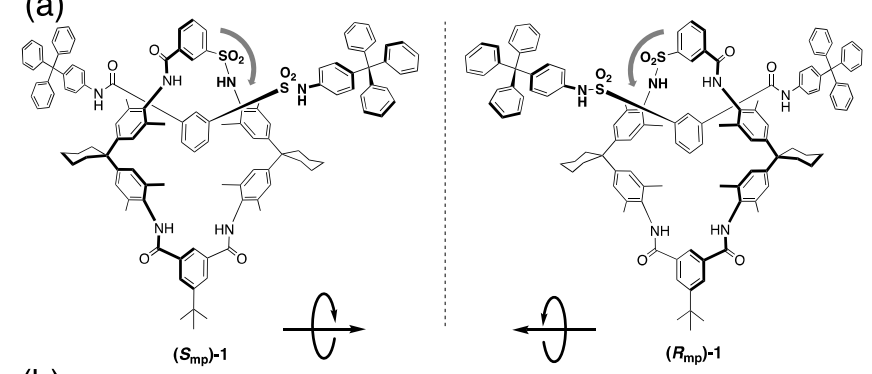

(1)
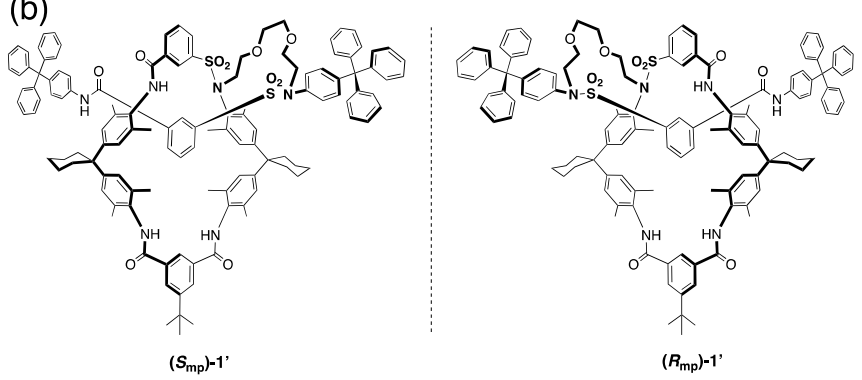

Figure 3. First synthesized and separated mechanically planar chiral rotaxanes: [2]rotaxane (a) and [1]rotaxane (b). The stereochemistry $S_{\mathrm{mp}}$ and $R_{\mathrm{mp}}$ is applied based on Goldup's method.

In their paper, the authors used the term "cycloenantiomeric" to describe the chirality of the rotaxanes according to Schill's nomenclature [16], which is based on the stereochemistry of cyclic peptides as reported by Prelog [4]. Based on the idea of cycloenantiomerism, Vögtle et al. determined the direction of the wheel (i.e., they applied the priority rule to the sulfonamide group in the wheel to decide the clockwise or anticlockwise). At that time, they did not discuss in $R$ or $S$ for absolute structure, but if Goldup's method is applied here, the enantiomers can be determined as $\left(S_{\mathrm{mp}}\right) \mathbf{- 1}$ and $\left(\boldsymbol{R}_{\mathrm{mp}}\right) \mathbf{- 1}$, respectively, as shown in Figure 3a. However, no one has ever succeeded in determining the absolute configuration of two actually separated enantiomer samples. To determine the absolute configuration, single crystal structure analysis or computational simulation would be required as the usual method, however it is still a difficult subject now.

Moreover, they also synthesized [3] rotaxanes, which have an additional wheel component compared with [2]rotaxane (Figure 4) [18]. As well as increasing the number of components, the number of stereoisomers is also increased. In [3]rotaxane, there are three stereoisomers that depend on different combinations of the orientations of the two wheels. As shown in Figure 4, two enantiomeric [3] rotaxanes with antiparallel orientated wheels $\left(\boldsymbol{R}_{\mathrm{mp}}, S_{\mathrm{mp}}\right)-2$ and $\left(S_{\mathrm{mp}}, \boldsymbol{R}_{\mathrm{mp}}\right)-2$ and the achiral meso-[3]rotaxane with parallel oriented wheels $\left(\boldsymbol{R}_{\mathrm{mp}}, \boldsymbol{R}_{\mathrm{mp} /} S_{\mathrm{mp}}, S_{\mathrm{mp}}\right)-2$ have been obtained in 1:1:2 in a statistical ratio. In these rotaxanes, the co-conformation is fixed by hydrogen bonds here, but a more complicated stereoisomerization system is expected, if it is possible to combine the co-conformational isomerization. Considering such a potentiality, these pioneering studies have contributed significantly to the creation of mechanostereoisomerization chemistry today.

The first attempt toward the asymmetric synthesis of a mechanically planar chiral rotaxane was reported by Takata et al. [19] (Scheme 1). They have already developed an effective rotaxane synthesis including an end-capping reaction of semi[2] rotaxane generated in situ from crown ether and dialkylammonium salt using a bulky anhydride and tributylphosphane catalyst. Therefore, they utilized a chiral catalyst instead of the tributylphosphane in hope of the mechanical chirality selective ester formation reaction. 


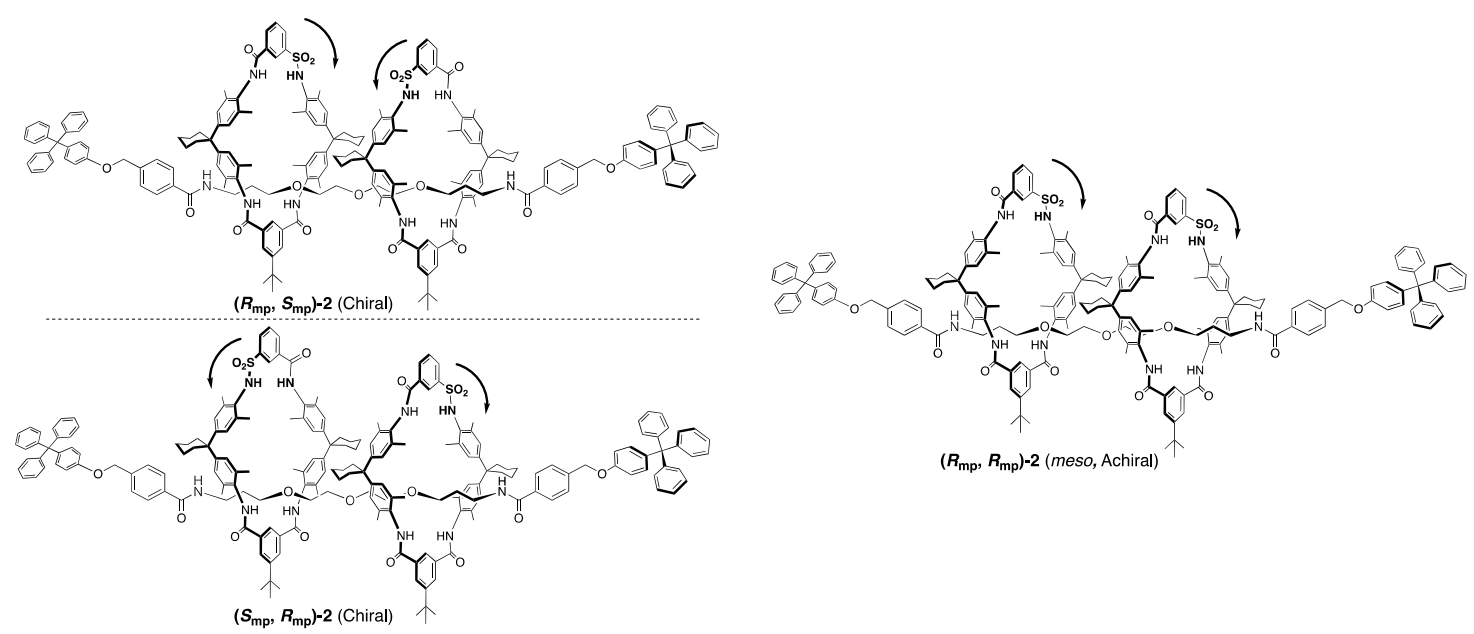

Figure 4. Mechanostereoisomers of [3]rotaxane composed of two wheels and a single axle component.

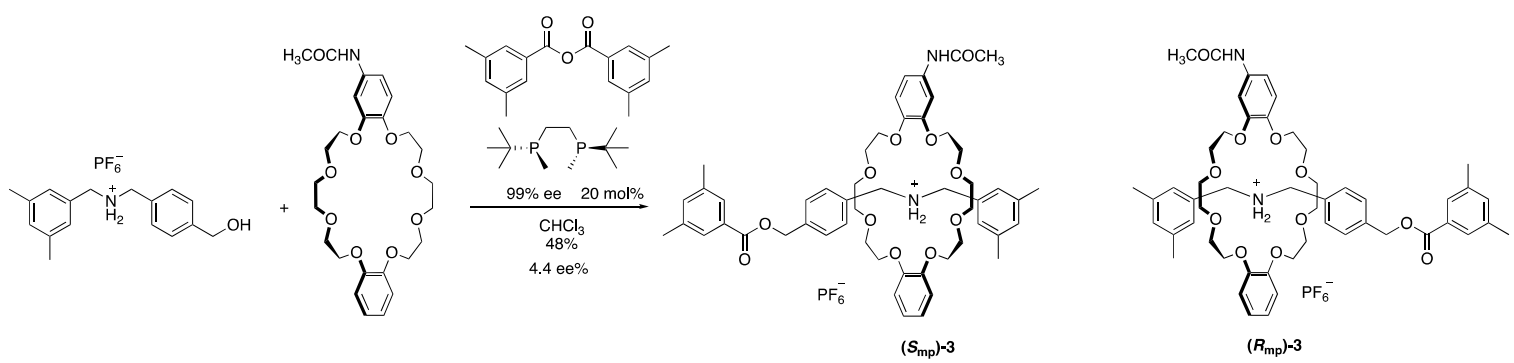

Scheme 1. Catalytic asymmetric synthesis of a mechanically planar chiral rotaxane.

As shown in Scheme 1, they utilized an optically active chiral ditopic phosphane as the catalyst and confirmed that the mechanically chiral [2] rotaxane 3 was obtained in $4.4 \mathrm{ee} \%$ by chiral stationary phase high performance liquid chromatography (HPLC) separation analysis. Unfortunately, the enantiomer excess was significantly low, but this is a meaningful first result. As it is surprising that these enantiomers are only different in the substitution position on the benzene ring of the wheel component, despite the fact that the components cannot be fixed in a specific relative arrangement during the end-cap reaction due to the dynamic nature of mechanical bonding. A typical strategy for the asymmetric synthesis is based on how to fix the spatial arrangement of the substrates around the chiral catalyst; some breakthroughs would be required toward effective asymmetric synthesis in dynamic mechanically chiral rotaxane.

Hirose reported a unique synthetic method to prepare mechanically chiral [2]rotaxanes utilizing enantiopure prerotaxanes [20] (Scheme 2).

Prerotaxane consists of a wheel component and a half-structured axle connected to the inner space of the wheel. After separation into enantiomerically pure prerotaxanes 4 by HPLC with a chiral stationary phase, these were converted to [2]rotaxanes $\left(R_{\mathrm{mp}} / S_{\mathrm{mp}}\right)-5$ by planar selective aminolysis of the ester group using 3,5-dinitrobenzylamine without chiral inversion or degradation.

As described above, chiral stationary phases have been indispensable in the optical resolution of mechanically chiral rotaxanes thus far; however, a breakthrough has recently been reported toward a more efficient separation of enantiomers. Thus, Goldup et al. achieved a practical optical resolution method by applying diastereomeric resolution to the synthesis of a mechanically chiral rotaxane (Scheme 3) [17]. 


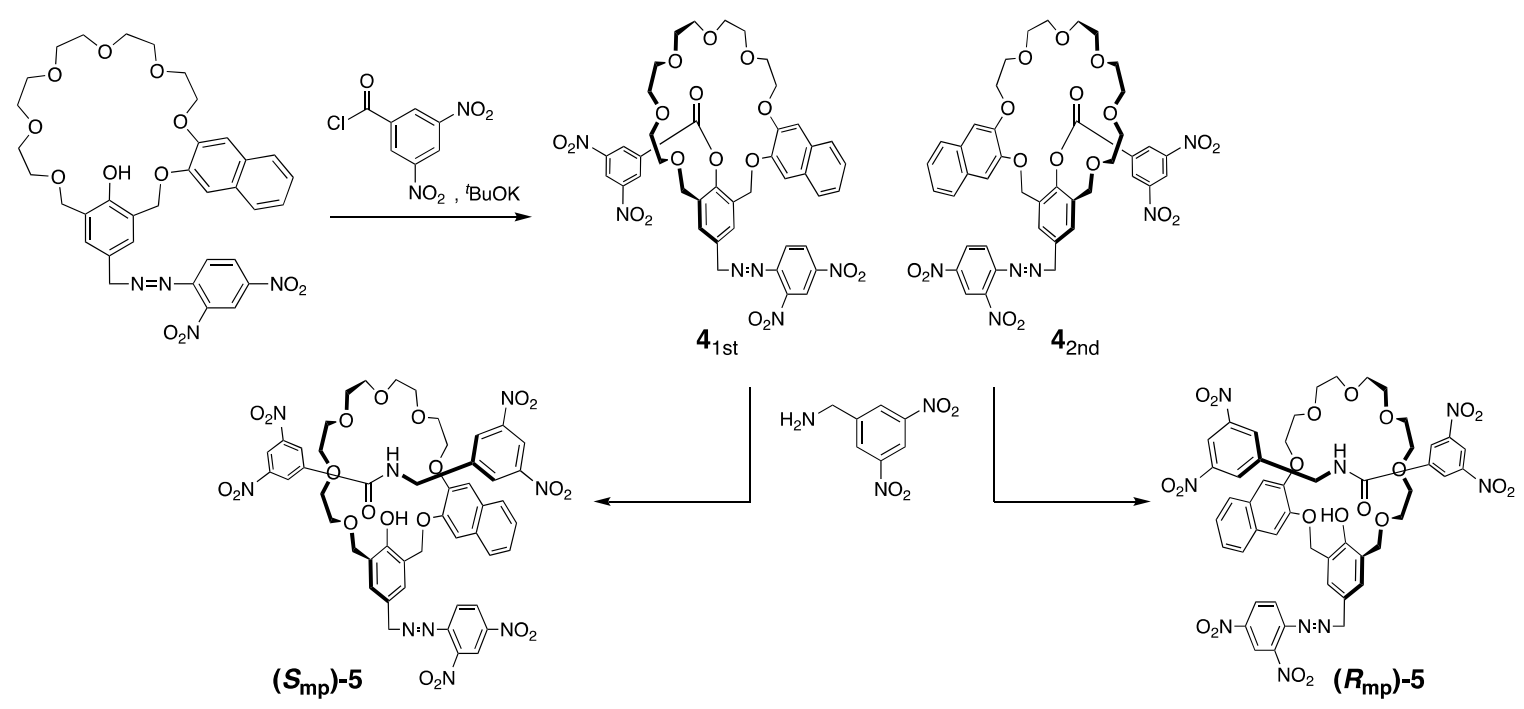

Scheme 2. Mechanically planar chiral [2]rotaxane synthesis from prerotaxane enantiomers. The structures of $\mathbf{4}_{1 \text { st }}$ (first elution) and $\mathbf{4}_{2 \text { nd }}$ (second elution) are tentatively determined configurations.

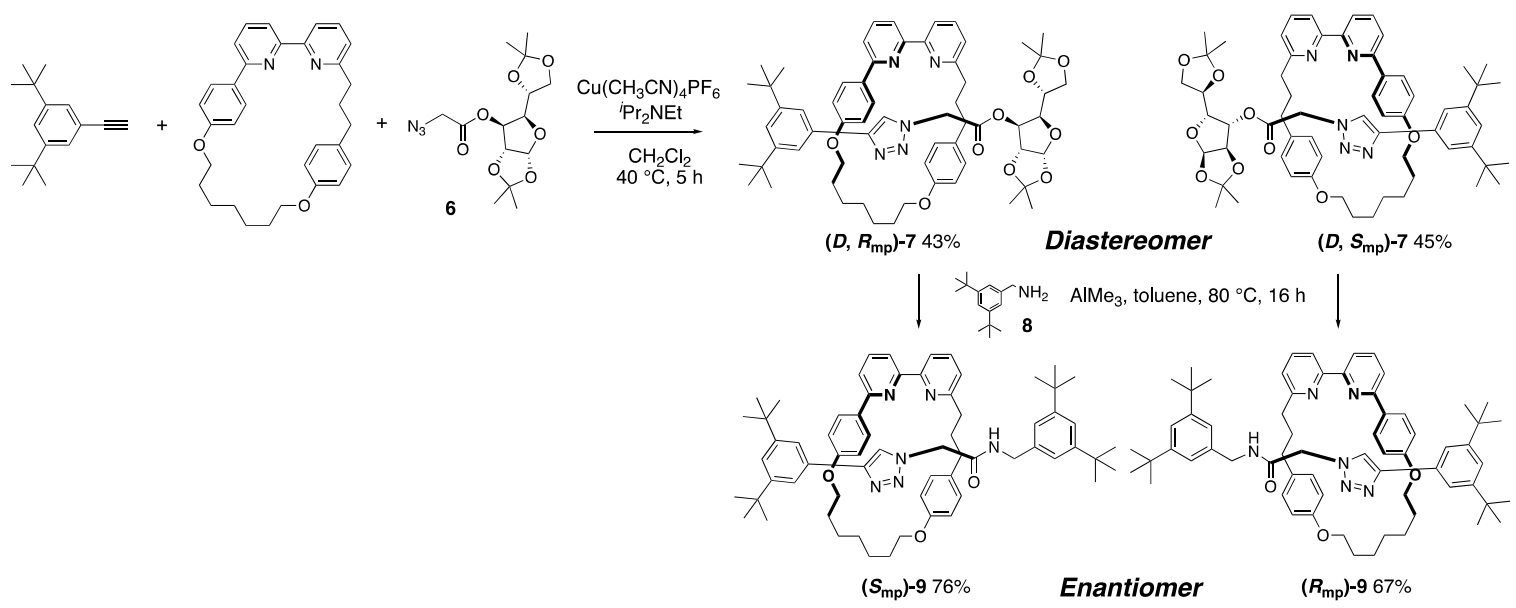

Scheme 3. Effective approach to an enantiomerically pure mechanically chiral rotaxane via practical resolution of the diastereomeric precursor.

A [2]rotaxane containing a $C_{\mathrm{s}}$ symmetrical wheel, an achiral half axle, and the enantiopure end-cap group 6 was synthesized by the copper-assisted azide-acetylene cycloaddition reaction. The diastereomers $\left(D, R_{\mathrm{mp}}\right)-7$ and $\left(D, S_{\mathrm{mp}}\right)-7$ were generated in a 1:1 ratio and separated by classical column chromatography using an achiral resin. The chiral end-cap was replaced with an achiral end-cap 8 via the substitution reaction to generate the mechanically chiral [2]rotaxane enantiomers $\left(S_{\mathrm{mp}}\right)-9$ and $\left(R_{\mathrm{mp}}\right) \mathbf{- 9}$, respectively. The configuration of $\mathbf{9}$ was changed from that of $\mathbf{7}$, however, this is because the direction of the axle determined according to the priority rule changed after the reaction, not the real stereoinversion of mechanical chirality. This catalytic ester-amide exchange reaction can achieve the stopper exchange reaction without dethreading of components to maintain the mechanical chirality.

Moreover, they increased the stereoselectivity of the diastereomeric mechanically planar rotaxane by changing the chiral end-cap group (Scheme 4) [21]. By using the readily available $\alpha$-amino acid-derived azide (S)-10 as the end-cap moiety, diastereomeric mechanically planar chiral [2]rotaxane $\left(S, R_{\mathrm{mp}} / S_{\mathrm{mp}}\right)-\mathbf{1 1}$ was obtained in a diastereomeric ratio of 98:2. The obtained diastereomers $\mathbf{1 1}$ can be transformed into an enantiomeric mechanically planar chiral [2] rotaxane $\left(\boldsymbol{R}_{\mathrm{mp}} / S_{\mathrm{mp}}\right) \mathbf{- 1 2}$ by converting the chiral end-cap to an achiral group, and the enantiomeric excess is unchanged. In their rotaxanes, 
it can be assumed that the short axle probably helps to reduce the co-conformational dynamic degree of freedom to enhance the reactivity of specific mechanical chirality.

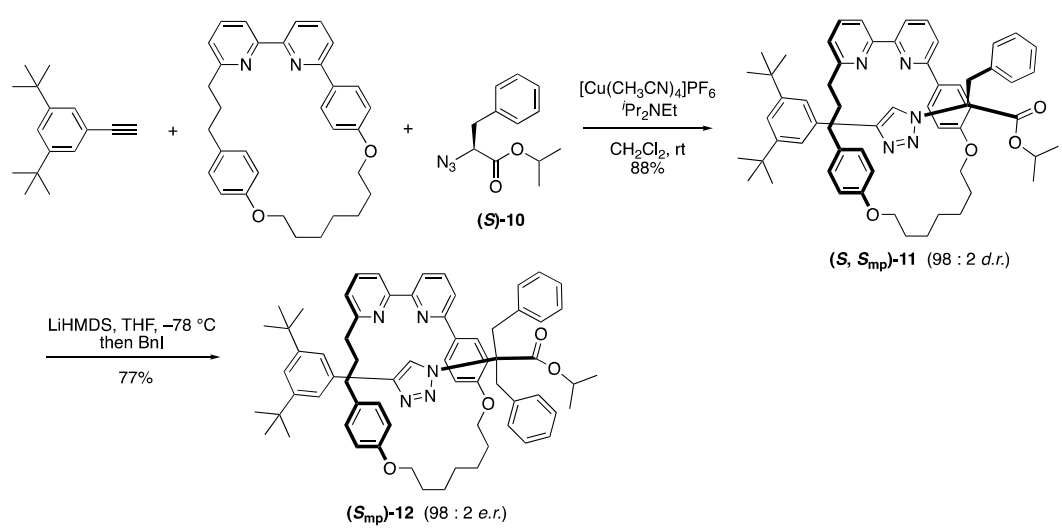

Scheme 4. Stereoselective synthesis of diastereomeric mechanically planar chiral [2]rotaxane and its transformation into enantiomeric [2]rotaxane.

Although, as has been described here, the synthesis and optical resolution of mechanically chiral rotaxanes are still under development, the intense ongoing research in this field will yield not only asymmetric synthesis, but also higher order and complicated topologically chiral rotaxanes in the not-so-distant future.

\subsection{Chiroptical Switching via Co-Conformational Interconversion of Mechanically Chiral Rotaxanes}

In this section, the rotaxane chirality is discussed from the perspective of co-conformation. Chiral information in MIMs can be transferred between components via mechanical bonds. In this context, Leigh and coworkers reported a chiroptical switching behavior in [2]rotaxane as the first example of through-space chirality transfer (Scheme 5) [22]. In [2]rotaxane 13, the wheel is located on the fumaramide moiety through hydrogen bonding, and no CD signal was observed. In contrast, after the photoisomerization of fumaramide, the wheel moves closer to the stereogenic center of the axle as 14, giving rise to a $C D$ signal. This chiroptical switch in the $C D$ spectrum occurred simultaneously with the reversible co-conformational change induced by photoisomerization, and suggests that the spatial arrangement of components around the mechanical bonding is fixed in the specific configuration in each co-conformer by hydrogen bonding between the components.
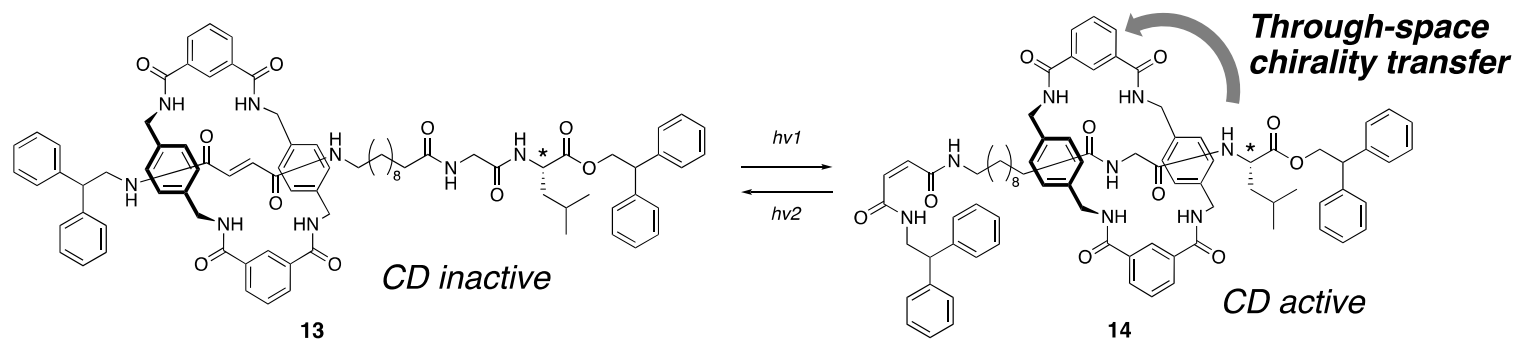

Scheme 5. Through-space chirality transfer of point chirality on the axle-to-wheel component.

Such a structure-constraining effect around the mechanical bonding also applied circularly polarized luminescence (CPL) control. Cuerva and Blanco designed a [2]rotaxane consisting of a wheel incorporating a 2,2'-bipyrene unit as the emissive part and an axle bearing point chiral stereogenic center nearby the sec-ammonium moiety (Scheme 6) [23]. This rotaxane is capable of on-off switching the CPL (i.e., the crown ether positioned on the sec-ammonium neighboring chiral stereogenic center induces CPL on (15), while the crown ether move to the triazolium moiety by deprotonation of ammonium makes CPL off (16)). In this way, the structure and chemical property of each component 
are deeply related to each other, and their intramolecular cooperative behavior makes it possible to transform the point chirality to axial chirality, depending on the co-conformational change.

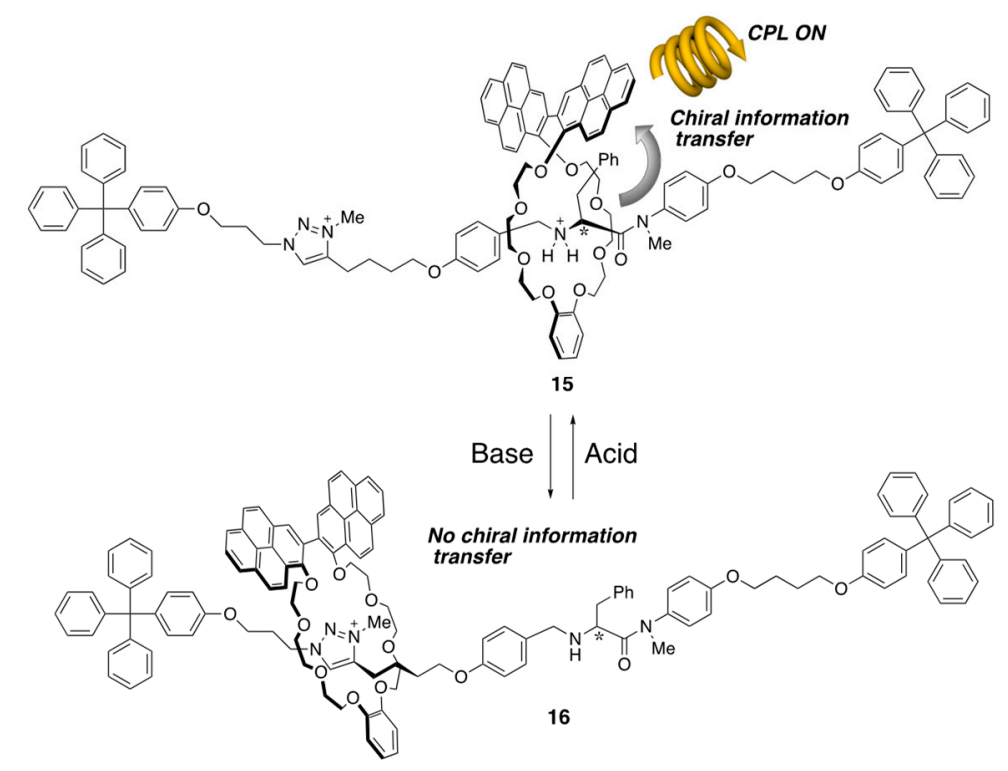

Scheme 6. On-off switching of CPL emission by controlling the through-space chirality transfer.

On the other hand, co-conformational chiroptical switching of mechanically planar chirality in rotaxanes was reported by Saito and coworkers (Scheme 7) [24].
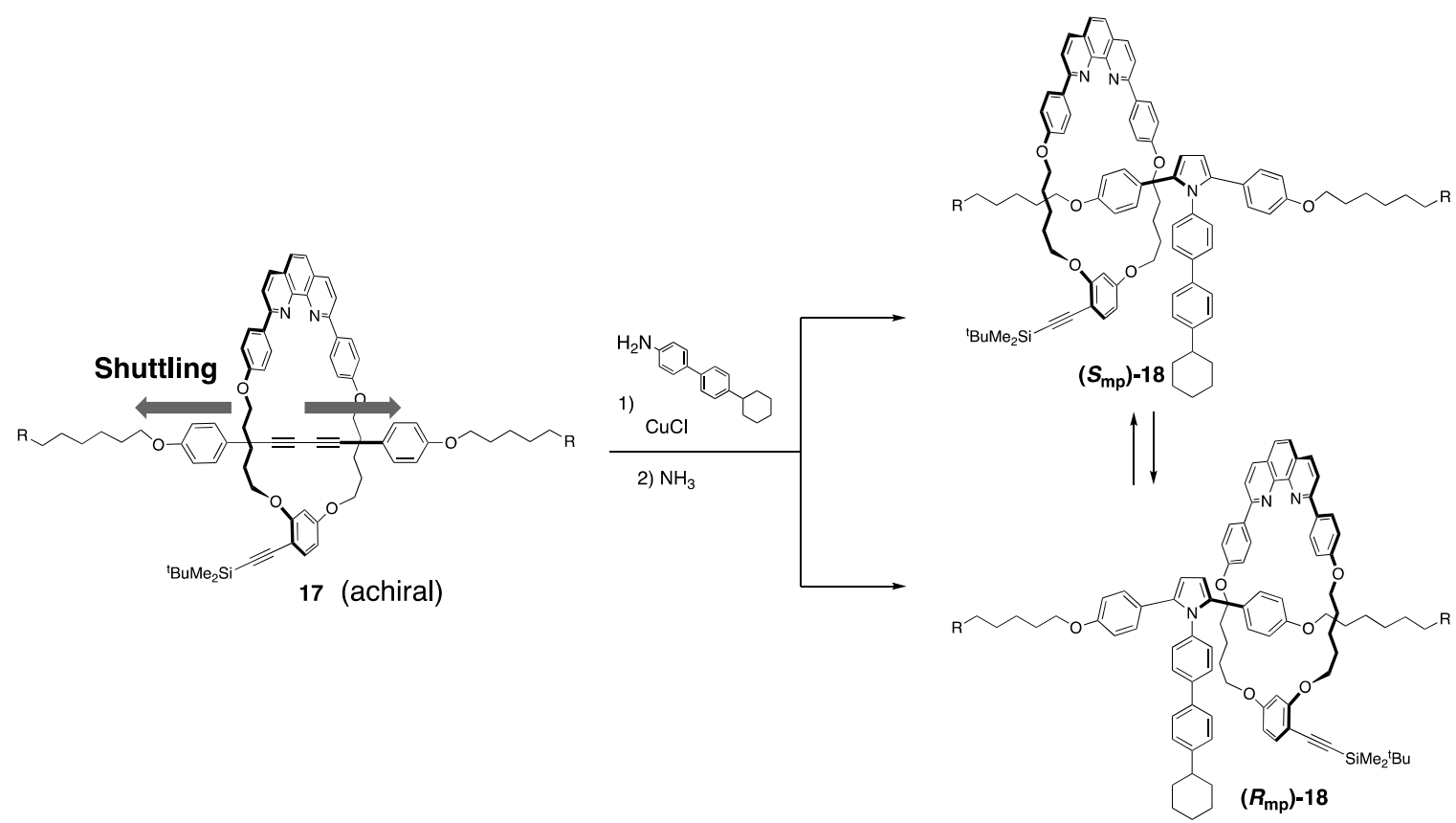

Scheme 7. Co-conformational mechanical chirality in [2]rotaxanes.

This kind of chirality is generated when a symmetrical axle is combined with a wheel with $C_{\mathrm{s}}$ symmetry. In other words, when the wheel is located at the center of the axle component, the molecule is prochiral (17), whereas when the wheel is located at a biased position (18), mechanical chirality is induced. Regardless of which side the wheel moves from the center of the axle, its shuttling energy is the same. Thus, if the partition group in the middle of the axle is sufficiently bulky, the enantiomers can be separated without racemization. In fact, the authors achieved the separation of enantiomerically 
pure [2]rotaxanes, which displayed a mirror image CD spectra. The relationship between these enantiomers involves a chiral inversion like "turning over the rubber gloves" $[25,26]$. A catenane with similar chirality was also reported by Sauvage et al. [25].

In rubber-glove-like chirality, there is no potential bias to determine the wheel's shuttling direction on the axle in general. Credi and Baroncini et al. achieved a one-directional selective shuttling by utilizing a diastereomeric salt system (Scheme 8) [27].

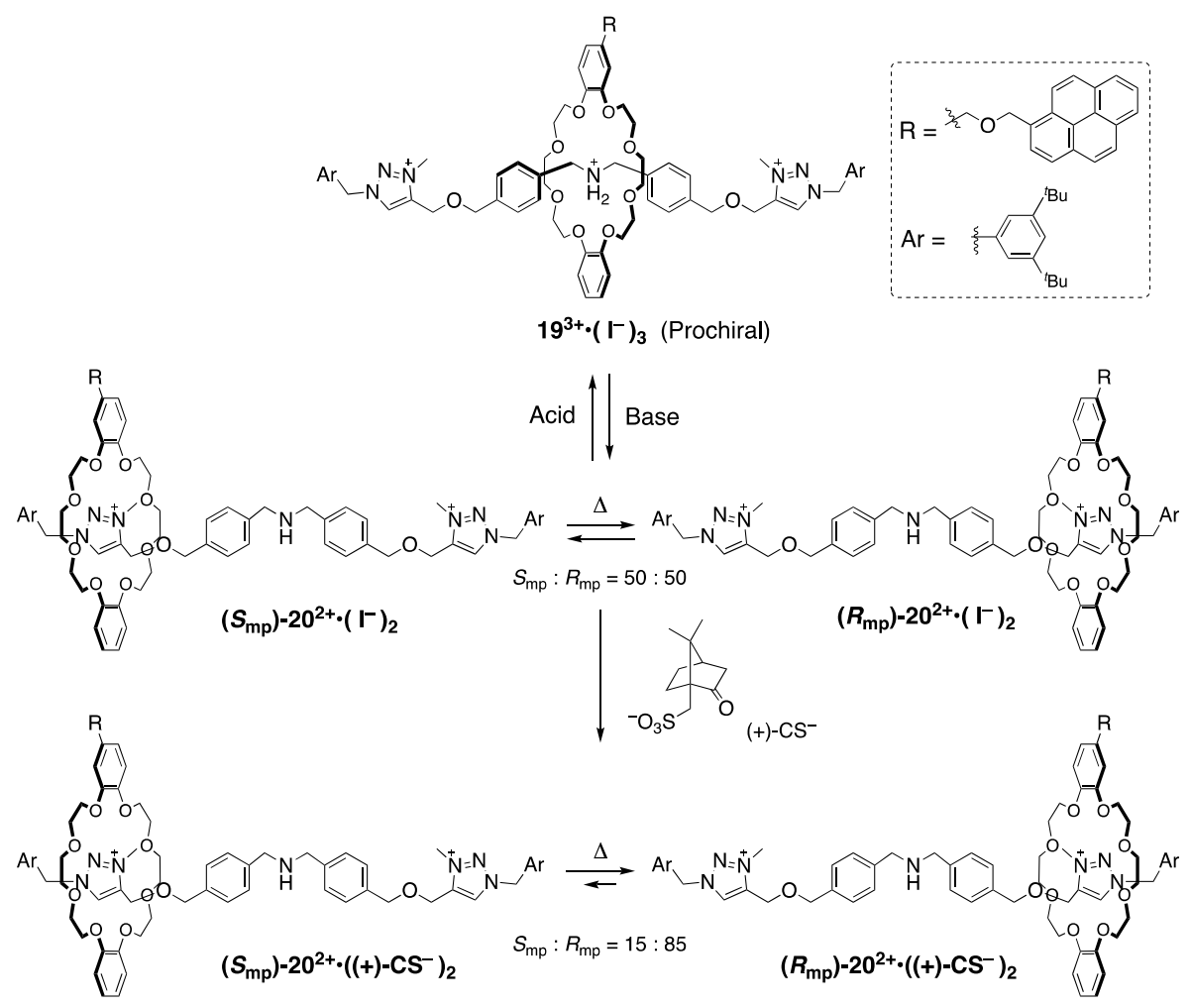

Scheme 8. Interconversion between two diastereomeric ion pairs containing a chiral anion with co-conformational mechanical chirality [27].

[2]Rotaxane 20, derived from prochiral [2]rotaxane 19, potentially has mechanical chirality, but these are dynamic racemic mixtures because the wheel component can move freely on the axle, having a symmetrical structure. The authors aimed to unbalance the populations of the wheel at the equivalent two triazolium stations by using an optically active counter anion such as the camphor sulfonate anion (CS) instead of achiral iodonium. As a result, they achieved an approximately 85:15 diastereomeric ratio. The authors suggested that the encircled triazolium site does not influence the site selectivity; the recognition between the wheel and another triazolium station that directly interacts with the optically active counter anion seems to be the primary reason for this selectivity. When the counter anion is exchanged with the iodonium ion instead of the optically active CS, the biased existence of diastereomers is canceled as a racemic mixture. Moreover, once the central amino group is protonated, the crown ether is localized at the prochiral position by strong hydrogen bonding, thus it becomes achiral rotaxane 19. These interconversions and inversions of the mechanical chirality are demonstrated in the solution state, so unfortunately, the separation of the diastereomers would be impossible in this system as there is no barrier to inhibit the racemization via shuttling motion.

Recently, Takata and Ishiwari achieved the chiral-achiral interconversion system [28]. As shown in Scheme 9, they derived mechanically chiral [2]rotaxane $\left(S_{\mathrm{mp}} / \boldsymbol{R}_{\mathrm{mp}}\right)$-22 from prochiral (achiral) [2]rotaxane $2 \mathbf{1}^{+} \cdot \mathbf{P F}_{\mathbf{6}}{ }^{-}$by N-ethylation of the nitrogen atom located at the symmetrical center of the axle. 


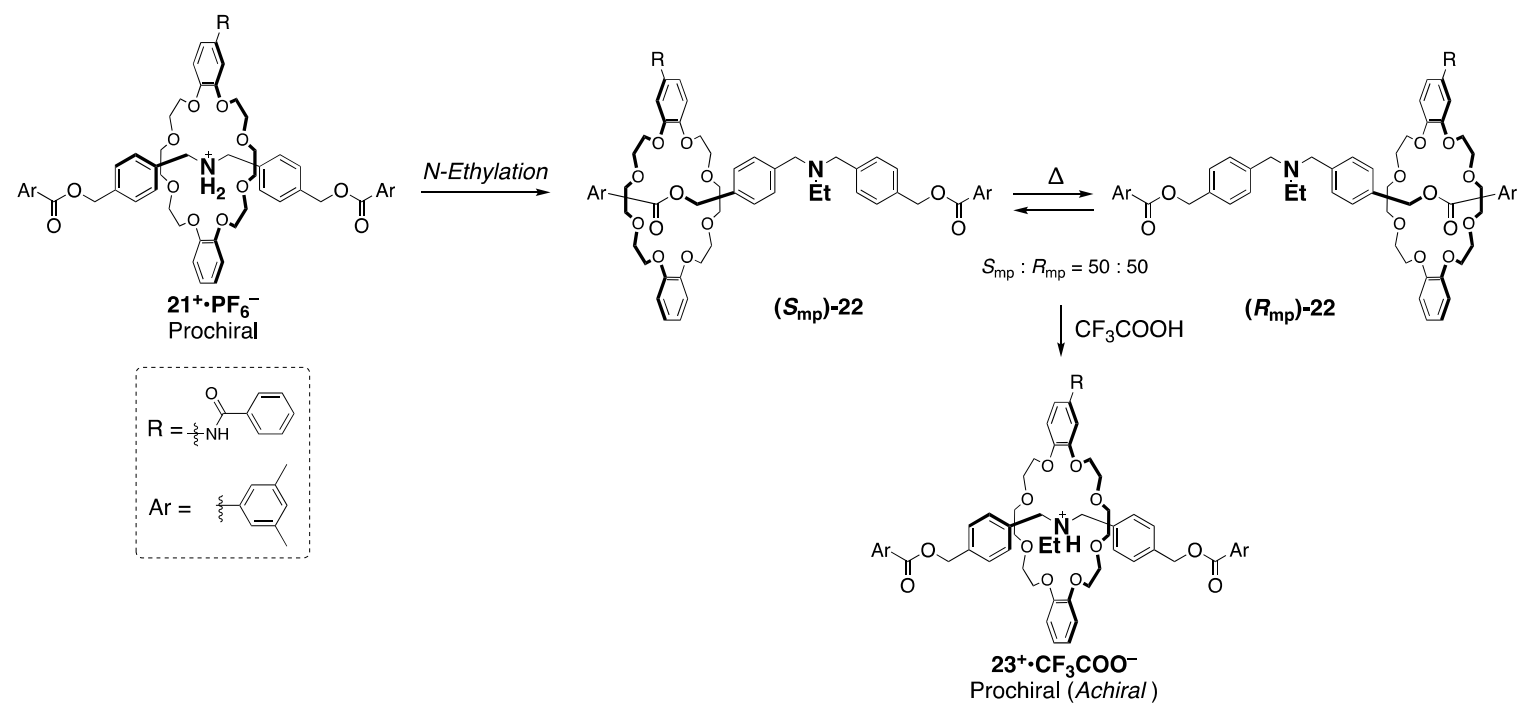

Scheme 9. Chiral-achiral reversible control of rotaxanes with a symmetrical axle [28].

Despite the N-ethyl group being size-complemental to the wheel, they achieved separation of the enantiomers in optical purities over $80 \mathrm{ee} \%$ by carrying out chiral HPLC resolution at a lower temperature than room temperature. In fact, the mirror imaged $\mathrm{CD}$ spectra were observed at $-10^{\circ} \mathrm{C}$ to support that these were enantiomers. The racemization behavior via the shuttling overcoming the central $\mathrm{N}$-ethyl group was also indicated by the $\mathrm{CD}$ decay at $0{ }^{\circ} \mathrm{C}$. By utilizing the protonation of the $\mathrm{N}$-ethyl group, [2]rotaxane 21 is also capable of being the achiral form $23^{+} \bullet \mathrm{CF}_{3} \mathrm{COO}^{-}$, not racemization. This chiral-achiral reversible interconversion is like the case in Scheme 8, however, setting up an appropriate barrier at the center of the axle would be the key to the further application of switchable mechanically chiral rotaxanes.

As indicated from these studies, the stereoinversion of mechanically chiral rotaxane by shuttling is similar to the chirality of axially chiral biphenyl compounds. This implies that the rubber-glove-like chirality of rotaxane is the spatio-temporal characteristics. In case of rotaxane, which is capable of stereoinverting, if some interaction is induced to fix the wheel on the center atom of the axle, the chirality disappears and becomes achiral. However, the ring components that are not covalently bonded on the center of the axle are still movable on the axle. Therefore, we cannot precisely distinguish the racemic state and prochiral state because the detected averaged structure is almost the same, irrespective of the varied degree of shuttling and circumrotation along the axle if the averaged structure coincides with the prochiral one.

Through these above-mentioned studies, it is considered that chiral rotaxanes potentially have chiroptical switching properties such as on-off switching or switching the handedness of enantiomers induced by various stimuli. The above-mentioned on-off switching of the CPL emission system in Scheme 6 is not attributed to the mechanical chirality, but is a highly suggestive study toward applications in optical devices with a chiroptical switching property. In the near future, if a method of controlling mechanical chirality involving co-conformation is established, a good example of CPL emission attributable to mechanical chirality will be achieved. It is expected that not only chiral achiral switching, but also stereo inversion, is possible, considering the chiroptical diversity due to the co-conformational diversity. Furthermore, several interesting application studies on dynamic helical polymers have been reported. These are discussed in the next section.

\subsection{Helical Structure Control by Chiral Rotaxanes}

Helical structure is a chiral structure universally found in polymers including biological macromolecules such as proteins, DNA, and RNA. The functions of these macromolecules are deeply related to their helical structure, and therefore the construction of well-ordered helical structures 
has attracted a great deal of attention. Polymers with a dynamic structure exhibit various physical properties such as crystallinity and glass transition temperature, which depend on their conformation of either a random coiled conformation or a highly ordered helical conformation. In this decade, significant advances have been made in the control of the structure of dynamic helical polymers and, recently, in the study of the application of their dynamic property as stimuli responsive chiral systems [29]. The chirality of a dynamic helical polymer is determined by various chiral factors such as catalyst, solvent, or interactive molecules as well as by the optically active group inside the polymer chain. More interestingly, the screw sense and helical pitch of the helical structure are affected by small changes in solvent polarity [30]. Thus, by controlling the dynamic helical structure of polymers, their chirality can be amplified from that of small or easily obtained chiral sources to higher order chirality. Another question of interest yet to be resolved is the prediction of the sense of a helical structure on a dynamic helical polymer from an enantiomerically pure chiral molecule that serves as a monomer, catalyst, or solvent. Although computational simulation might help in this regard, this is a challenging task because the structure usually depends on multiple environmental stimuli. Despite the large number of examples of polymers with a switchable helical structure, those exhibiting controlled reversible helicity inversion in one pot are still scarce. In this context, Takata et al. achieved a rational control of the helical structure of polyacetylene with chiral [2]rotaxanes (Figure 5) [31].

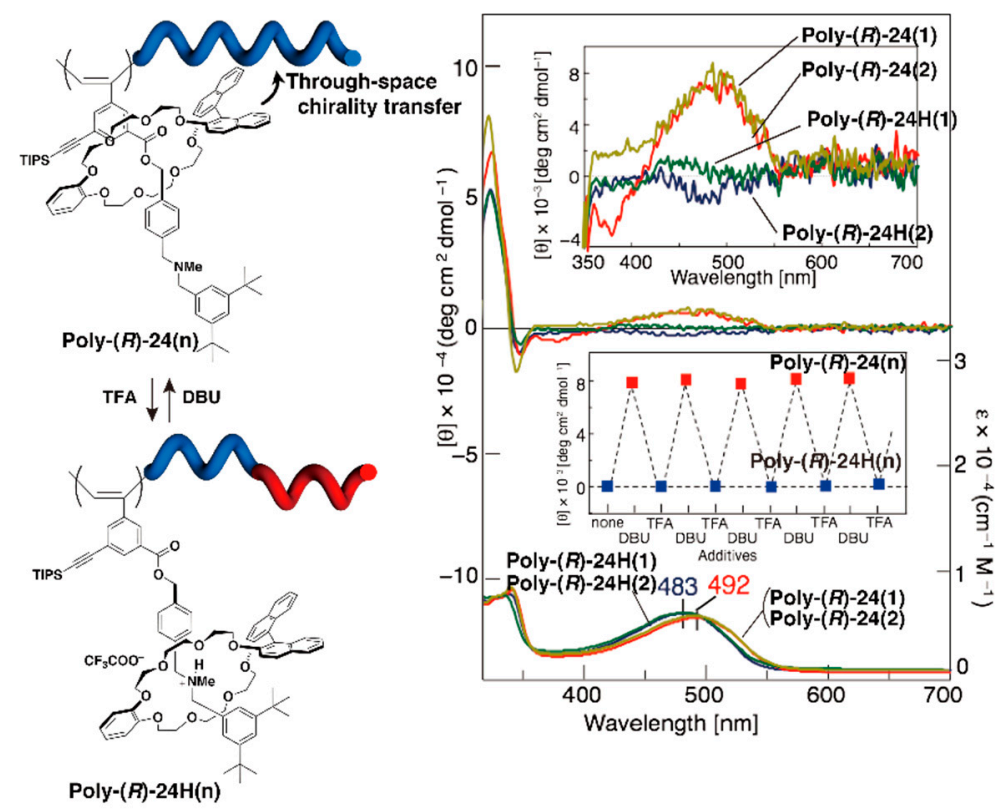

Figure 5. Control of reversible helicity inversion triggered by chiral rotaxane side chain co-conformational switching, and the corresponding circular dichroism (CD) and UV spectra (chloroform, $0.14 \mathrm{mM}, 293 \mathrm{~K}$ ). The insets are the expanded CD spectra (350-500 nm, top) and the reversible change in CD intensity $(500 \mathrm{~nm})$ with various additives (bottom).

Polyacetylene is a typical dynamic helical polymer that has prompted several studies on the control of its higher order structures. As shown in Figure 5, the side chain-type polyrotaxane poly-(R)-24 was prepared by the polymerization of chiral [2]rotaxane with an acetylene-terminated axle and a crown ether containing an optically active binaphthyl unit. In this system, the distance between the chiral wheels and the polymer main chain is precisely tuned by the reversible co-conformational change of rotaxane in the polymer side chain. The CD and ultraviolet-visible absorption (UV-Vis) spectra of poly-(R)-24, which has a tert-amine-bearing axle, show a Cotton effect attributable to the polymer main chain absorption region, supporting the induction of the single-handed helical structure on the polymer backbone. After protonation of the tert-amine to give poly-(R)-24H, the Cotton effect completely disappeared. This indicates that the polymer main chain becomes a random helix because the chiral 
wheels are fixed by hydrogen bonding at the ammonium moiety, which is sufficiently far from the polymer main chain. Such a change in the helical structure of the polymer main chain occurs in one pot in response to acid or base treatment, indicating that the helical structure is induced with good reproducibility. This result shows the potential of "through-space chirality transfers and amplification" from the mechanically linked chiral wheel to the polymer backbone by utilizing the intramolecular chiroptical switching ability of the rotaxane side chain.

More recently, Takata et al. achieved the induction of a single-handed helical structure from the mechanical chirality of the rotaxane side chains (Figure 6) [32].

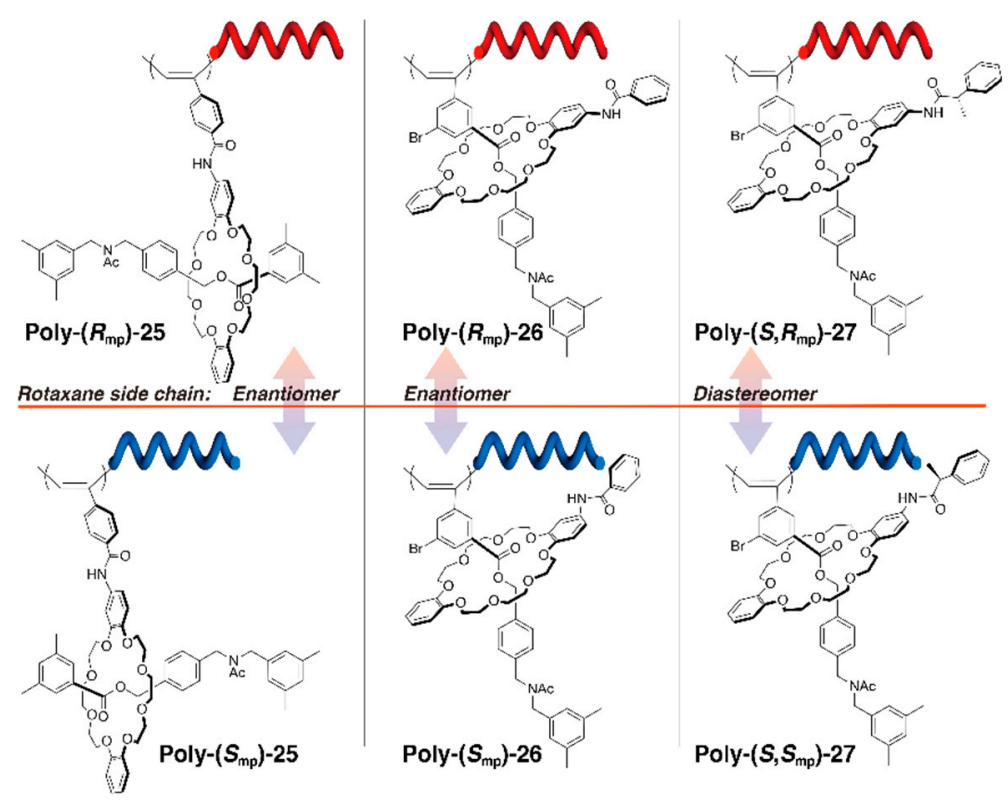

Figure 6. Control of the polymer helical structure by mechanically chiral rotaxane side chains.

They synthesized three types of polyrotaxanes-poly- $\left(R_{\mathrm{mp}} / S_{\mathrm{mp}}\right)-25$, poly- $\left(R_{\mathrm{mp}} / S_{\mathrm{mp}}\right)-26$, and poly-(S, $\left.R_{\mathrm{mp}} / S, S_{\mathrm{mp}}\right)$-27-with different rotaxane side chains depending on the mechanical chirality of rotaxanes of how to link with the polymer backbone. The polymer shown on the left of Figure 6 has an enantiomerically pure mechanically chiral rotaxane side chain linked to the polymer backbone via the wheel, whereas the polymer shown in the center of the figure is linked via the axle. In both cases, the polymer obtained from each enantiomer exhibited a mirror image CD spectrum in the absorption region of the main chain for the opposite sense of the helical structure. The structural difference between the enantiomers are quite small, and it is interesting to determine which of the adjacent carbon atoms of the benzene ring are substituted. When viewed from the polymer backbone, it is noteworthy that the chirality of the helical structure is induced even though the wheel components are not chiral and are not fixed in a specific conformation because of the flexibility of the mechanical bond. Furthermore, an additional point of chirality on the wheel component in the side chain does not influence the helicity of the polymer, as in $\left(S, R_{\mathrm{mp}} / S, S_{\mathrm{mp}}\right)-27$. This result clearly indicates that mechanically chiral rotaxanes, despite having a dynamic nature, can be used as efficient chiral sources, sometimes even in a more effective manner than classical chirality in which the configuration is fixed.

It has also been confirmed that the reversible helical chirality inversion depends on both temperature and solvent. Although the role of mechanical chirality in the induction of single-handed helical structures is not fully understood, this inversion in the polymer probably stems from the occurrence of a conformational change due to the movement of mechanical bonds as a result of environmental stimuli. These results introduce an important concept that opens the door to a new chiral chemistry and could result in the development of novel applications. 


\section{Conclusions and Outlook}

Nowadays, rotaxane is an easily accessible molecule that possesses a variety of potential applications as an innovative small molecular device, catalyst, and molecular sensing. However, the fundamental aspects of rotaxanes such as their stereochemistry are not yet fully understood at the present time. The determination of the absolute configuration of mechanically chiral rotaxanes is still a challenging task. Considering that this issue remains unsolved even for simple rotaxanes, the problem becomes more complicated when increasing the number of components, which leads to more complex isomers including conformational isomers. Therefore, further fundamental study is absolutely required. Furthermore, the application to the catalysis and molecular sensing of mechanically chiral molecules should be helpful in understanding how the mechanical chirality works to other environmental molecules, along with the development of the asymmetric synthetic method. In addition, through study including molecular assemblies, we will be able to gain deeper insights into the properties and functions of the mechanical bonds and its chirality from the viewpoints of both chemical and physical consideration. These application studies through collaboration across fields would bring great benefits to the fundamental research of MIMs. We hope that chemists from various fields including supramolecular chemistry will have interest in mechanical chirality and develop new aspects of chemicals, materials, and related science areas.

Author Contributions: Writing—original draft preparation and visualization, K.N.; Writing—review and editing, T.T. All authors have read and agreed to the published version of the manuscript.

Funding: This research was funded by the JSPS KAKENHI (No. 19K05417), the Naito Foundation and the CREST program (JST, JPMJCR1522).

Acknowledgments: K.N. acknowledges funding from JSPS KAKENHI (No. 19K05417) and The Naito Foundation. T.T. and K.N. thank the CREST program (JST, JPMJCR1522) for financial support.

Conflicts of Interest: The authors declare no conflicts of interest.

\section{References}

1. Bruns, C.J.; Stoddart, J.F. The fundamentals of making mechanical bonds. In The Nature of the Mechanical Bond: From Molecules to Machines, 1st ed.; John Wiley \& Sons, Inc.: Hoboken, NJ, USA, 2017; Chapter 2; pp. 55-268.

2. Bruns, C.J.; Stoddart, J.F. Molecular switches and machines with mechanical bonds. In The Nature of the Mechanical Bond: From Molecules to Machines, 1st ed.; John Wiley \& Sons, Inc.: Hoboken, NJ, USA, 2017; Chapter 6; pp. 555-733.

3. Frisch, H.L.; Wassermann, E. Chemical topology. J. Am. Chem. Soc. 1961, 83, 3789-3795. [CrossRef]

4. Prelog, I.V.; Gerlach, H. Cycloenantiomerism and cyclodiasteromerism I. Helv. Chim. Acta 1964, 47, $2288-2294$.

5. Gerlach, H.; Ovchinnikov, Y.A.; Prelog, V. Cycloenantiomerism and cyclodiastereomerism II. Cycloenantiomeric cyclohexaalanyls and cyclodiglycyltetraalanyls. Helv. Chim. Acta 1964, 47, 2294-2302. [CrossRef]

6. Kaida, Y.; Okamoto, Y.; Chambron, J.-C.; Mitchell, D.K.; Sauvage, J.-P. The separation of optically active copper (I) catenates. Tetrahedron Lett. 1993, 34, 1019-1022. [CrossRef]

7. Jäger, R.; Händel, M.; Harren, J.; Rissanen, K.; Vögtle, F. Chemistry with rotaxanes: Intra- and intermolecularly covalently linked rotaxanes. Liebigs Ann. 1996, 1996, 1201-1207. [CrossRef]

8. Yamamoto, C.; Okamoto, Y.; Schmidt, T.; Jäger, R.; Vögtle, F. Enantiomeric resolution of cycloenantiomeric rotaxane, topologically chiral catenane, and pretzel-shaped molecules: Observation of pronounced circular dichroism. J. Am. Chem. Soc. 1997, 119, 10547-10548. [CrossRef]

9. Bruns, C.J.; Stoddart, J.F. The stereochemistry of the mechanical bond. In The Nature of the Mechanical Bond: From Molecules to Machines, 1st ed.; John Wiley \& Sons, Inc.: Hoboken, NJ, USA, 2017; Chapter 5; pp. 471-554.

10. Vignon, S.A.; Stoddart, J.F. Exploring dynamics and stereochemistry in mechanically-interlocked compounds. Collect. Czech. Chem. Commun. 2005, 70, 1493-1576. [CrossRef]

11. Leigh, D.A.; Perez, E.M. Dynamic chirality: Molecular shuttles and motors. Top. Curr. Chem. 2006, 265, 185-208. 
12. Neal, E.A.; Goldup, S.M. Chemical consequences of mechanical bonding in catenanes and rotaxanes: Isomerism, modification, catalysis and molecular machines for synthesis. Chem. Commun. 2014, 50, 5128-5142. [CrossRef]

13. Evans, N.H. Chiral catenanes and rotaxanes: Fundamentals and emerging applications. Chem. Eur. J. 2018, 24, 3101-3112. [CrossRef]

14. Pairault, N.; Niemeyer, J. Chiral mechanically interlocked molecules-applications of rotaxanes, catenanes and molecular knots in stereoselective chemosensing and catalysis. Synlett 2018, 29, 689-698.

15. Jamieson, E.M.G.; Modicom, F.; Goldup, S.M. Chirality in rotaxanes and catenanes. Chem. Soc. Rev. 2018, 47, 5266-5311. [CrossRef] [PubMed]

16. Schill, G. Catenanes, Rotaxanes, and Knots; Academic Press: New York, NY, USA, 1971.

17. Bordoli, R.J.; Goldup, S.M. An efficient approach to mechanically planar chiral rotaxanes. J. Am. Chem. Soc. 2014, 136, 4817-4820. [CrossRef] [PubMed]

18. Schmieder, R.; Hubner, G.; Seel, C.; Vögtle, F. The first cyclodiastereomeric [3]rotaxane. Angew. Chem. Int. Ed. 1999, 38, 3528-3530. [CrossRef]

19. Makita, Y.; Kihara, N.; Nakakoji, N.; Takata, T.; Inagaki, S.; Yamamoto, C.; Okamoto, Y. Catalytic asymmetric synthesis and optical resolution of planar chiral rotaxane. Chem. Lett. 2007, 36, 162-163. [CrossRef]

20. Hirose, K.; Ukimi, M.; Ueda, S.; Onoda, C.; Kano, R.; Tsuda, K.; Hinohara, Y.; Tobe, Y. The asymmetry is derived from mechanical interlocking of achiral axle and achiral ring components-syntheses and properties of optically pure [2]rotaxanes. Symmetry 2018, 10, 20. [CrossRef]

21. Jinks, M.A.; Juan, A.; Denis, M.; Fletcher, C.J.; Galli, M.; Jamieson, E.M.G.; Modicon, F.; Zhang, Z.; Goldup, S.M. Stereoselective synthesis of mechanically planar chiral rotaxanes. Angew. Chem. Int. Ed. 2018, 45, 14806-14810. [CrossRef]

22. Bottari, G.; Leigh, D.A.; Perez, E.M. Chiroptical switching in a bistable molecular shuttle. J. Am. Chem. Soc. 2003, 125, 13360-13361. [CrossRef]

23. David, D.H.G.; Casares, R.; Cuerva, J.M.; Campana, A.G.; Blanco, V. A [2]rotaxane-based circularly polarized luminescence switch. J. Am. Chem. Soc. 2019, 141, 18064-18074. [CrossRef]

24. Mochizuki, Y.; Ikeyatsu, K.; Mutoh, Y.; Hosoya, S.; Saito, S. Synthesis of mechanically planar chiral rac-[2]rotaxanes by partitioning of an achiral [2]rotaxane: Stereoinversion induced by shuttling. Org. Lett. 2017, 19, 4347-4350. [CrossRef]

25. Chambron, J.-C.; Sauvage, J.-P.; Mislow, K.; Cian, A.D.; Fischer, J. A [2]catenane and a [2]rotaxane as prototypes of topological and Euclidean molecular "rubber gloves". Chem. Eur. J. 2001, 7, 4085-4096. [CrossRef]

26. Walba, D.M. Chemical Applications of Topology and Graph Theory. In Studies in Physical and Theoretical Chemistry; King, R.B., Ed.; Elsevier: Amsterdam, The Netherlands, 1983; Volume 8, pp. 17-32.

27. Corra, S.; de Vet, C.; Groppi, J.; La Rosa, M.; Silvi, S.; Baroncini, M.; Credi, A. Chemical on/off switching of mechanically planar chirality and chiral anion recognition in a [2]rotaxane molecular shuttle. J. Am. Chem. Soc. 2019, 141, 9129-9133. [CrossRef] [PubMed]

28. Ishiwari, F.; Takata, T. Rotaxanes with Dynamic Mechanical Chirality: Systematic Studies on Synthesis, Optical Resolution, Racemization, and Chiral-ProchiralInterconversion. Chemrxiv 2019. [CrossRef]

29. Yashima, E.; Ousaka, N.; Taura, D.; Shimomura, K.; Ikai, T.; Maeda, K. Supramolecular helical systems: Helical assemblies of small molecules, foldamers, and polymers with chiral amplification and their functions. Chem. Rev. 2016, 116, 13752-13990. [CrossRef] [PubMed]

30. Nagata, Y.; Nishikawa, T.; Suginome, M.; Sato, S.; Sugiyama, M.; Porcar, L.; Martel, A.; Inoue, R.; Sato, N. Elucidating the solvent effect on the switch of the helicity of poly(quinoxaline-2,3-diyl)s: A conformational analysis by small-angle neutron scattering. J. Am. Chem. Soc. 2018, 140, 2722-2726. [CrossRef]

31. Ishiwari, F.; Nakazono, K.; Koyama, Y.; Takata, T. Rational control of a polyacetylene helix by a pendant rotaxane switch. Chem. Commun. 2011, 47, 11739-11741. [CrossRef]

32. Ishiwari, F.; Nakazono, K.; Koyama, Y.; Takata, T. Induction of single-handed helicity of polyacetylenes using mechanically chiral rotaxanes as chiral sources. Angew. Chem. Int. Ed. 2017, 56, 14858-14862. [CrossRef]

(C) 2020 by the authors. Licensee MDPI, Basel, Switzerland. This article is an open access article distributed under the terms and conditions of the Creative Commons Attribution (CC BY) license (http://creativecommons.org/licenses/by/4.0/). 